\title{
ON THE EXISTENCE OF BERTRAND PAIRS
}

by

\section{A. M. d'Azevedo Breda, F. J. Craveiro de Carvalho and Bernd Wegner}

In this note we introduce the Bertrand Group for a curve. While there is no mention of this group in it, [6] contains a statement which would mean that for a simple, closed, twisted curve that group would be trivial. However there appears to be a flaw in the proof given there.

Here we are able to show that for a simple, twisted curve that group is either trivial or $Z_{2}$. If this latter group can occur we do not know. We also show that for some classes of curves the non-triviality of that group forces the curve to be plane.

1. In what follows $X$ will stand for $R$ or $S^{1}$ and we will be dealing with smooth space curves, that is, $C^{\infty}$ immersions $f: X \rightarrow R^{3}$. Moreover the curvature $k_{f}$ of $f$ is assumed not to vanish. This way we have a well defined Frenet-Serret frame $\left(T_{f}, N_{f}, B_{f}\right)$ everywhere. We will not assume parametrization by arc-length and the velocity of $f$ will be denoted by $v_{f}$.

Two curves $f, g: X \rightarrow R^{3}$ are Bertrand mates [3] if, for every $x \in X$, the principal normal lines of $f$ and $g$ at $x$ coincide.

Definition 1 : The Bertrand Group $B(f)$ of $f: X \rightarrow R^{3}$ is the subgroup of Diff $(X)$ formed by the diffeomorphisms $\delta: X \rightarrow X$ such that $f$ and $f \circ \delta$ are Bertrand mates.

Proposition 1 : Let $f: X \rightarrow R^{3}$ be a smooth curve with non-vanishing curvature. Then $B(f)$ is

a) cyclic of finite order if $X=S^{1}$.

b) trivial or not finite if $X=R$.

Proof: Denote by $A_{1}^{3}$ the open Grassmannian of affine lines in $R^{3}$ and define $\widetilde{N}: X \rightarrow A_{1}^{3}$, where $\widetilde{N}(x)$ is the principal normal line at $x$. Since we are assuming non-vanishing curvature $\widetilde{N}$ is an immersion. This fact implies that the action $\phi: B(f) \times X \rightarrow X$, with $\phi(\delta, x)=\delta(x)$, is properly discontinuous. 
In fact let $\delta \in B(f)$ and suppose that $x \in X$ is such that $\delta(x)=x$. Since $\widetilde{N}$ is an immersion there is an open neighbourhood $U$ of $x$ such that $\widetilde{N} \mid U$ is injective. Then, for $y \in U \cap \delta^{-1}(U), \delta(y)=y$. Therefore the fixed point set $\Delta$ of $\delta$ is open. Since $\Delta$ is also closed it follows that either $\delta$ has no fixed points or is the identity. Consequently the action of $B(f)$ on $X$ is free.

Furthermore if $U \cap \delta(U) \neq \emptyset$ then $\delta$ is the identity and $B(f)$ acts in a properly discontinuous way. Hence the projection $p: X \rightarrow X / B(f)$ is a covering projection and $\pi_{1}(X / B(f), p(x)) / p_{*}\left(\pi_{1}(X, x)\right) \approx B(f)[5]$.

If $X=S^{1}$ then $X / B(f)$ is diffeomorphic to $S^{1}$ and it follows that $B(f)$ is cyclic of finite order.

Assume now that $X=R$ and that $B(f)$ is finite. Then $X / B(f)$ is either $R$ or $S^{1}$. Since $B(f) \approx \pi_{1}(X / B(f))$ it follows that it must be trivial. $\bowtie$

If $\delta \in B(f)$ then it follows that $\|f(x)-f(\delta(x))\|$ does not depend on $x$.

Proposition 2 : Let $\delta \in B(f)$ be such that $\|f(x)-f(\delta(x))\|>0$. Then the torsion $\tau_{f}$ never vanishes or vanishes everywhere.

Proof: There exist constants $c, c_{1}, c_{2}$ such that, for every $x$,

$$
f(\delta(x))=f(x)+c N_{f}(x) \text { and } T_{f}(\delta(x))=c_{1} T_{f}(x)+c_{2} B_{f}(x) .
$$

Now use the Frenet-Serret formulas to conclude that, for every $x$,

$$
c \tau_{f}(x)=\left(c_{2} \delta^{\prime}(x) v_{f}(\delta(x))\right) / v_{f}(x) .
$$

Then either $c_{2}=0$ which implies that $\tau_{f}$ vanishes everywhere or $\tau_{f}$ never vanishes.

$\infty$

For some important classes of curves the non-triviality of the Bertrand group implies severe restrictions on the curves.

Proposition 3: Let $f: S^{1} \rightarrow R^{3}$ be a simple curve. If $f$ is transnormal or spherical and $B(f)$ is non-trivial then $f$ is a plane curve.

Proof: If $f$ is spherical then its torsion $\tau_{f}$ vanishes somewhere. This can be seen by considering $h: S^{1} \rightarrow R$, given by $h(x)=\left(B_{f}(x) \mid f(x)-a\right)$, where $a$ is the centre of the sphere containing $f\left(S^{1}\right)$. Naturally $h$ has critical points and these are points where the torsion vanishes. 
If $f$ is transnormal then it is known [4] that every normal affine plane meets the image of $f$ in exactly two points. If $\delta \in B(f)$ is non-trivial then, for the normal affine plane at $x \in S^{1}$, those two points are $f(x)$ and $f(\delta(x))$. It follows that $T_{f}(\delta(x))=-T_{f}(x)$. From the proof of Proposition 2 above we see that $\tau_{f}$ vanishes everywhere.

$\infty$

Also let us consider now a smooth map $f: S^{1} \rightarrow R$ and define $F: S^{1} \rightarrow R^{3}$ by $F(x)=(x, f(x))$. A short calculation shows that the torsion $\tau_{F}$ vanishes somewhere. Therefore we have

Proposition 4: Let $f: S^{1} \rightarrow R$ be a smooth map and $F: S^{1} \rightarrow R^{3}$ be defined by $F(x)=(x, f(x))$. If $B(F)$ is not trivial then $f$ is constant.

We can also use Proposition 2 to establish

Proposition 5: Let $f: S^{1} \rightarrow R^{3}$ be a simple curve such that its image is symmetrical with respect to a point $p \notin f\left(S^{1}\right)$. If $B(f)$ is not trivial then $f\left(S^{1}\right)$ is a circle with $p$ as the centre.

Proof: Assume without loss of generality that the centre of symmetry $p$ is the origin $O$ in $R^{3}$. Then there is a diffeomorphism $\alpha: S^{1} \rightarrow S^{1}$ such that $A \circ f=f \circ \alpha$, where $A$ is the reflection with respect to the origin. Since we have $\tau_{f}(\alpha(t))=\tau_{f \circ \alpha}(t)=\tau_{A \circ f}(t)=-\tau_{f}(t)$ it follows that $\tau_{f}$ vanishes everywhere and $f$ is a plane curve.

For plane curves, as we will mention again below, to say that $B(f)$ is non-trivial is equivalent to say that $f$ is self-parallel. Also, for plane curves, self-parallelism is equivalent to transnormality [1]. The result then follows by Proposition $8, \S 4$ in [2].

$\infty$

If $\tau_{f}$ vanishes everywhere then to say that $B(f)$ is non-trivial is equivalent to say that $f$ is self-parallel and self-parallel curves were studied in [1] and [7]. In the case of embeddings (injective immersions which are homeomorphisms onto the image) $B(f)$ is either $Z_{2}$ or trivial according as $X$ is $S^{1}$ or $R$.

2. It is easy to obtain examples of non-injective curves with cyclic or infinite Bertrand groups. Let us concentrate now on simple curves with nonvanishing torsion. 
Proposition 1: Let $f: X \rightarrow R^{3}$ be a simple curve with non-vanishing torsion. Then $B(f)$ is trivial if $X=R$ and it is trivial or $Z_{2}$ if $X=S^{1}$.

Proof: Assume that $X=R$. If $B(f)$ is not trivial choose two distinct elements from $B(f) \backslash\left\{i d_{R}\right\}$. We have then two distinct and non-zero constants $a_{1}, a_{2}$ such that, for $x \in S^{1}$,

$$
f\left(\delta_{1}(x)\right)=f(x)+a_{1} N_{f}(x), f\left(\delta_{2}(x)\right)=f(x)+a_{2} N_{f}(x) .
$$

If follows [3] that the reparametrizations by arc-length of $f$ are circular helices and we reach a contradiction for the Bertrand group of $f$ would then be trivial.

Similar arguments allow us to conclude that $B(f)$ is either trivial or $Z_{2}$ when $f$ is a simple closed curve. We only have to argue replacing $\delta_{1}, \delta_{2}$ by $\delta, \delta^{2}=\delta \circ \delta$, where $\delta$ is the generator of $B(f)$.

$\bowtie$

Remark: Let $f: S^{1} \rightarrow R^{3}$ be a simple curve and assume that $B(f)$ is $Z_{2}$. If $\delta$ is the generator of $B(f)$ then there are constants $c_{1}, c_{2}$ such that, for $x \in S^{1}$, we have

$$
\begin{aligned}
& T_{f}(\delta(x))=c_{1} T_{f}(x)+c_{2} B_{f}(x), \\
& B_{f}(\delta(x))=c_{2} T_{f}(x)-c_{1} B_{f}(x) .
\end{aligned}
$$

That is, $T_{f}(\delta(x))$ and $B_{f}(\delta(x))$ are obtained from $T_{f}(x)$ and $B_{f}(x)$ by a reflection in the vector subspace $\left\langle T_{f}(x), B_{f}(x)>\right.$. This appears to be the point that has been overlooked in [6] when coming to the conclusion that the vectors $T_{f}(\delta(x)), T_{f}(x)$ are linearly dependent.

\section{References}

1. F. J. Craveiro de Carvalho and S. A. Robertson, Self-Parallel curves, Math. Scand. 65 (1989), 67-74.

2. F. J. Craveiro de Carvalho and Bernd Wegner Diametrical submanifolds, Periodica Mathematica Hungarica 40 (2000), 1-11. 
3. Chuan-Chih Hsiung, A first couse in differential geometry, John Wiley \& Sons, 1981.

4. M. C. Irwin, Transnormal circles, J. London Math. Soc. 42 (1967) $545-552$.

5. C. Kosniowski, A first course in algebraic topology, Cambridge University Press, 1980.

6. Giacomo Saban, Barbier's theorem through self-mated closed Bertrand curves, Simon Stevin 57 (1983), 37-45.

7. B. Wegner, Self-parallel and transnormal curves, Geom. Dedicata $\mathbf{3 8}$ (1991), 175-191.
A. M. d'Azevedo Breda
Departamento de Matemática
Universidade de Aveiro
Portugal
E-mail: ambreda@mat.ua.pt
F. J. Craveiro de Carvalho
Departamento de Matemática
Universidade de Coimbra
Portugal
E-mail: fjcc@mat.uc.pt
Bernd Wegner
Fachbereich Mathematik
TU Berlin
Germany
E-mail: wegner@math.tu-berlin.de 